\title{
Leadless Pacemaker Implantation in a 4-year-old, 16-kg Child
}

\author{
Arjun Mahendran ${ }^{1}$, Sara Bussey ${ }^{1}$, and Philip Chang ${ }^{1}$ \\ ${ }^{1}$ University of Florida Health
}

April 27, 2020

\begin{abstract}
Leadless pacemakers have an accepted role with demonstrable benefit in adults. In contrast, implant and follow-up experience in pediatric patients is quite limited. We present our implant experience in a 4-year-old for treatment of high-grade AV block. Implant considerations in small patients and follow-up assessment of the vessel used for implantation are discussed.
\end{abstract}

\section{Case Report}

A 4-year-old male $\left(16.1 \mathrm{~kg}, 0.67 \mathrm{~m}^{2}\right)$ infant heart transplant recipient presented with unexplained syncope. Historically, despite aggressive immunosuppression, he experienced chronic antibody-mediated rejection and markedly elevated panel reactive antibodies precluded re-transplantation candidacy. Long-term treatment has prioritized palliative care and maximization of quality of life.

Inpatient telemetry captured episodes of second-degree, Mobitz type II AV block with symptoms of tiredness and dizziness (Fig 1). Episodes lasted for several minutes with spontaneous resolution. Heart block was considered the mechanism for syncope and a sequela of chronic graft rejection. Permanent pacing was indicated and traditional and non-traditional pacing options were considered. Implications of each approach including potential impact on vascular access, relative ease of future endomyocardial biopsies, and postprocedure recovery were discussed. Collective agreement between the parents, transplant team, cardiac surgeons, and electrophysiologist was reached to pursue leadless pacemaker implantation. With the recent availability of the FDA-approved Micra ${ }^{\mathrm{TM}} \mathrm{AV}$ pacemaker at our institution, this was selected for its potential benefit in addressing the patient's specific pacing indication (ie. heart block).

Conventional right femoral venous implantation was performed under general anesthesia. Ultrasound-guided venous access was obtained and a 6-French (Fr) sheath was inserted. Contrast injection through the sheath was performed to assess femoral and iliac vein caliber. Given the vessels' capacity for distension, slow and serial dilation was performed over an exchange length stiff wire with a series of dilators culminating in the dilator of the Micra introducer sheath (IS), followed by insertion of the complete IS set (27-Fr outer diameter). Insertion of the IS proceeded smoothly without resistance.

The Micra delivery catheter (DC) was prepped and advanced through the IS. To cross the tricuspid valve (TV), active flexion of the DC was applied with counterclockwise torque. Mid-septal implantation was initially attempted, however adequate septal orientation of the delivery cup could not be achieved. Furthermore, given the dimensions of the chamber at this location, mid-septal deployment was felt to potentially obstruct the TV apparatus proximally and the RV outflow and pulmonary valve superiorly (Fig $2 \mathrm{~A}$ ). Therefore, an apical septal location was pursued. The TV was crossed again more inferiorly. Clockwise torque was applied after crossing the valve to direct the DC tip toward the apical septum (Fig 2B). The device was deployed with at least 2 splines exhibiting appropriate fixation. Testing demonstrated acceptable functional values ( $\mathrm{R}$ waves $>10 \mathrm{mV}$, pacing threshold $0.63 \mathrm{~V}$ at $0.24 \mathrm{~ms}$, impedance $570 \mathrm{ohms}$ ). Transesophageal imaging confirmed the implant location and showed no pericardial effusion or significant tricuspid interference. The anchoring tether was removed with preservation of device position (Fig 2C). Following DC withdrawal and 
before IS removal, an 0-Ethibond pursestring suture was placed around the skin entry site for closure and hemostasis. The site was covered with a pressure dressing. Post-implant interrogation showed preserved functional values and reasonable accelerometer-derived signals corresponding to atrial contraction (Fig 3).

Right lower extremity (LE) vascular ultrasound performed 2 days post-implant showed a near-occlusive thrombus in the common femoral vein (CFV). The pressure dressing and skin suture were removed on postimplant day \#4 without any evidence of bleeding or hematoma. Given the luminal thrombus, subcutaneous heparin was initiated without bleeding recurrence. Follow-up assessment at 6 weeks post-implant showed normal device function and interval reduction in thrombus size with normal vessel flow pattern on follow-up LE scanning. The patient had returned to normal activities without syncope recurrence.

\section{Discussion}

To our knowledge, this case represents the smallest pediatric patient implanted with the Micra leadless pacemaker and the first implanted with the newest model with VDD functionality. The patient's pacing indication, coupled with avoidance of more invasive implant procedures and complications with leads (transvenous or epicardial) and subcutaneous device pockets, made leadless pacing preferable, especially in light of his long-term palliative care strategy.

Published implant and follow-up experience with leadless pacemakers in pediatric patients has been limited, with fewer than 20 total implants. The largest single-center cohort included 9 patients (median age 13 years and median weight $37 \mathrm{~kg}$ ). ${ }^{1}$ A conventional femoral venous approach has generally been taken, though all patients were $>11$ years of age and $>30 \mathrm{~kg}$ in weight. ${ }^{1-3}$ Alternatively, Gallotti et al and Cortez presented separate cases using right internal jugular venous access in smaller patients. ${ }^{4-5}$ Post-implant assessment of the vessels used for implantation has not been previously reported.

Our case demonstrates several important considerations with a conventional femoral venous approach in very small patients. Slow and serial vessel dilation should be performed. The IS itself with its hydrophobic outer surface advanced very smoothly even in the small vessel used in our case. Catheter flexion and device positioning in the RV cavity were easily accomplished. The application of counterclockwise torque with flexion was extremely effective for TV crossing. A mid-septal implant location with Micra may not be ideal in small patients owing to the smaller RV chamber size and potential risk of inflow and outflow interference. In addition, adequate septal orientation of the DC tip may not be achievable at this more proximal location on the septum. Post-implant development of a near-occlusive thrombus in the CFV, while not entirely unexpected, was unfortunate. It is encouraging that venous patency was preserved with interval reduction of the luminal clot on short-term anticoagulation therapy.

While we hope the VDD pacing capability of Micra AV can be useful for our patient's primary pacing indication, its use in young patients may be limited due to its relatively low programmable upper tracking rate $(115 \mathrm{bpm})$. Given an anticipated low pacing burden and the sporadic nature of Mobitz II AV block in our patient, we were unable to record significant VDD pacing over the short follow-up period. However, manual testing of atrial sensing demonstrates reasonable signals corresponding to atrial contraction and we would anticipate that synchronous pacing can be effectively applied within programmable parameters.

Device retrievability is of particular interest in pediatric patients and has been successfully performed up to 4 years post-implant. ${ }^{6}$ Given the smaller sizes of RV chambers in pediatric patients, the Micra's footprint may be proportionately substantial and abandonment of a battery-depleted Micra may limit space for new device implantation. Retrievability was not considered to be a limiting factor in our case given the device's anticipated long battery longevity in comparison to the patient's long-term prognosis.

In summary, conventional femoral Micra implantation is feasible even in small sized patients. The lower limits for age and size for safe and effective Micra pacemaker implantation have not been established but appear smaller than previously published. Assessment of implant vessel size and its capacity for dilation and large sheath accommodation must be performed. Vessel and cardiac chamber size can pose limitations to implantation in smaller patients. Synchronous pacing expands the potential use of leadless pacing even 
in pediatric patients, though upper tracking rates may be a limitation.

\section{References}

1. Breatnach CR, Dunne L, Al-Alawi K, Oslizlok P, Kenny D, Walsh KP. Leadless Micra Pacemaker Use in the Pediatric Population: Device Implantation and Short-Term Outcomes. Pediatr Cardiol 2019, Epub: https://doi.org/10.1007/s00246-019-02277-y.

2. Tejman-Yarden S, Nof E, Beinart R, Ovadia N, Goldshmit Y, Buber J, Yonath H, Keizman E, Glikson M. Leadless Pacemaker implantation in a Pediatric Patient with Prolonged Sinus Pauses. Pediatr Cardiol 2018;39:844-847.

3. McCanta AC, Morchi GS, Tuozo F, Berdjis F, Starr JP, Batra AS. Implantation of a Leadless Pacemaker in a Pediatric Patient with Congenital Heart Disease. HeartRhythm Case Rep 2018;4:506-509.

4. Gallotti RG, Biniwale R, Shannon K, Russell M, Moore JP. Leadless Pacemaker Placement in an 18-Kilogram Child: Procedural Approach and Technical Considerations. HeartRhythm Case Rep 2019;5:555-558.

5. Cortez D. Innovative Implantation of a Leadless Pacemaker in a $19 \mathrm{~kg}$ Paediatric Patient via the Right Internal Jugular Vein. Europace 2019;21:1542.

6. Kiani S, Merchant FM, El-Chami MF. Extraction of a 4-year-old Leadless Pacemaker with a Tine-based Fixation. HeartRhythm Case Rep 2019;5:424-425.

\section{Figures:}

Figure 1: Telemetry-captured second-degree, Mobitz II AV block (transient complete AV block shown). The patient had normal 1:1 AV conduction before and following the block event.

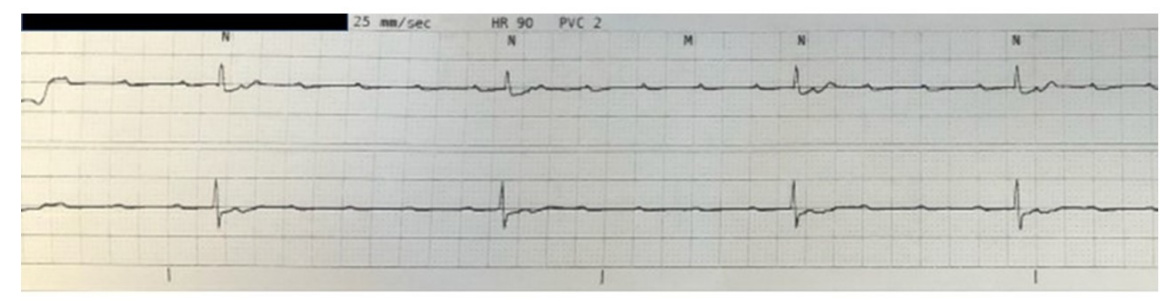

Figure 2A-C: A) Attempted mid-septal implantation: The DC cup extended toward the outflow tract and pulmonary valve (black arrow). B) Apical septal implantation: Contrast opacification seen at the septal RV apex (black arrow). C) Anterior-posterior fluoroscopic projection demonstrating the final implant position of the Micra AV device. 


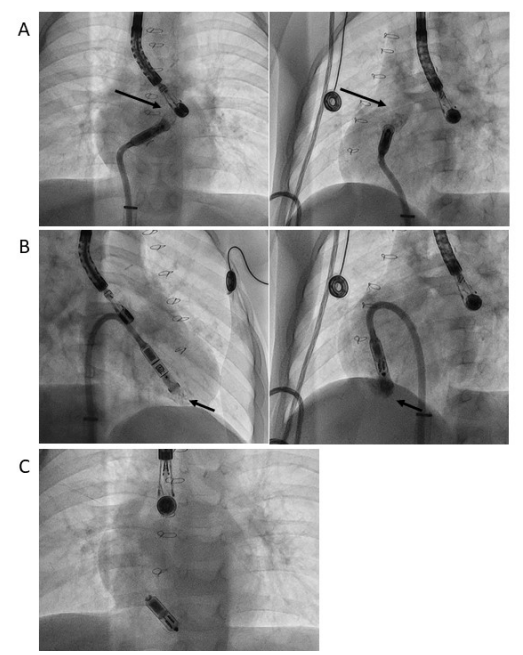

Figure 3: Post-implant interrogation with assessment of accelerometer signals: Sharp A1 and rounded A2 signals correspond to tricuspid valve (A1) and pulmonary valve (A2) closure during ventricular contraction. The A3 signal corresponds to passive ventricular filling. The A4 signal corresponds to atrial contraction.

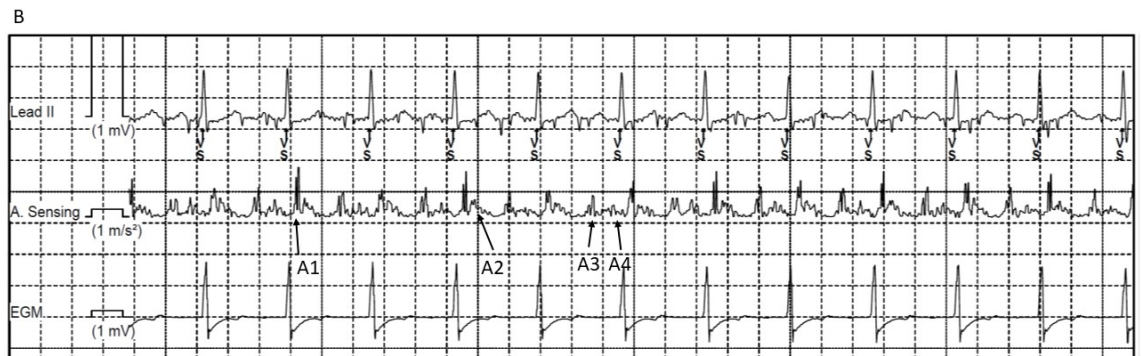

